\title{
Observation of Stimulated Emission of Radiation by Relativistic Electrons in a Spatially Periodic Transverse Magnetic Field*
}

\author{
Luis R. Elias, William M. Fairbank, John M. J. Madey, H. Alan Schwettman, and Todd I. Smith \\ Department of Physics and High Energy Physics Laboratory, Stanford University, Stanford, California 94305 \\ (Received 15 December 1975)

\begin{abstract}
Gain has been observed for optical radiation at $10.6 \mu \mathrm{m}$ due to stimulated radiation by a relativistic electron beam in a constant spatially periodic transverse magnetic field. A gain of $7 \%$ per pass was obtained at an electron current of $70 \mathrm{~mA}$. The experiments indicate the possibility of a new class of tunable high-power free-electron lasers.
\end{abstract}

We have observed the amplification of infrared radiation by relativistic free electrons in a constant, spatially periodic, transverse magnetic field. The experiment was conducted in the W. W. Hansen High Energy Physics Laboratory using an electron beam from the superconducting linear accelerator. The experiment was performed by sending the electron beam through the periodic field and measuring the gain and absorption coefficients for $10.6-\mu \mathrm{m}$ radiation sent through the field parallel to the electron beam axis.

Radiation emitted within the periodic field is properly termed magnetic bremsstrahlung. Radiation can also be absorbed via the process of inverse bremsstrahlung. The physical effect which makes amplification possible is the difference in wavelength between emission and absorption. The wavelength for emission is slightly longer than the wavelength for absorption. While the difference in wavelength is small, the rates for stimulated emission and absorption are large and net useful gain is predicted at wavelengths to below $1000 \AA$.

The stimulated emission of bremsstrahlung in a spatially periodic magnetic field was analyzed by Madey and co-workers. ${ }^{1,2}$ The physics is related to the problem of stimulated Compton scattering ${ }^{3}$ which has been analyzed by Driecer, ${ }^{4}$ Pantell, Soncini, and Puthoff, ${ }^{5}$ Sukhatme and Wolff, ${ }^{6}$ and Kroll. ${ }^{7}$ Other free-electron amplifiers have also been proposed. Hirshfield, Bernstein, and Wachte $1^{8}$ proposed and developed the cyclotron maser in which a relativistic electron beam moved through a uniform axial field, and Granatstein and co-workers ${ }^{9,10}$ at the Naval Research Laboratory have exploited stimulated magnetoRaman scattering to generate high-power submillimeter radiation.

The theory for stimulated bremsstrahlung predicts a correlation between the line shape for spontaneous radiation and the gain. Measurements were therefore made of both the spontane- ous radiation and the gain. The experimental apparatus is shown schematically in Fig. 1. The periodic magnetic field was generated by a superconducting right-hand double helix with a $3.2-\mathrm{cm}$ period and a length of $5.2 \mathrm{~m}$. The helix was wound around a 10.2-mm-i.d. evacuated copper tube which enclosed the interaction region. The field due to the helix was transverse and rotated in the plane normal to the axis with the period of winding. A 1-kOe axial guide field was generated by a solenoid wound over the helical magnet.

The electron beam and the infrared radiation were steered to pass through the magnet on the axis. Radiation from a pulsed transverse-excitation-atmospheric $\mathrm{CO}_{2}$ laser was focused to a 3.3$\mathrm{mm}$ waist at the entrance to the interaction region to excite the $\mathrm{EH}_{11}$ wave-guide mode of the 10.2$\mathrm{mm}$ copper tube. Calculations indicate that this configuration couples $98 \%$ of the energy in the incident Gaussian beam to the $\mathrm{EH}_{11}$ mode. ${ }^{11}$

Radiation emerging from the tube was focused on a fast Santa Barbara Research Corporation copper-doped germanium detector. The electron beam from the superconducting accelerator was bunched and the interaction between the electrons and optical radiation modulated the radiation at the $1300-\mathrm{MHz}$ accelerator operating frequency. The fractional modulation is equal to the gain per pass. The amplitude and phase of the $1300-\mathrm{MHz}$ modulation were recovered by mixing the detec-

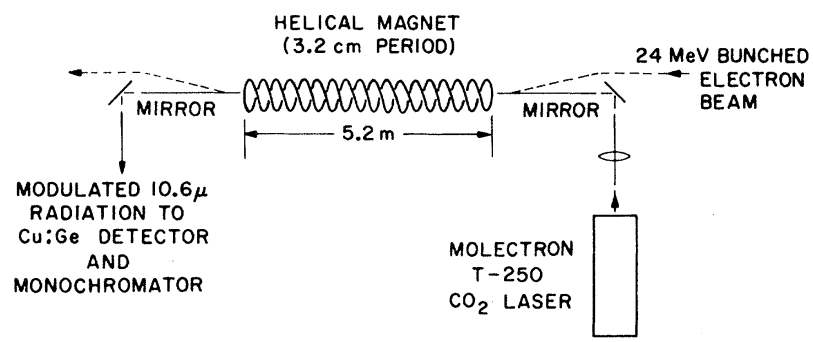

FIG. 1. Experimental setup. The electron beam was magnetically deflected around the optical components on the axis of the helical magnet. 
tor output with a signal derived from the accelerator master oscillator. The local oscillator phase was set to maximize the signal due to spontaneous radiation by the electron beam. The relative response of the detector to $1300-\mathrm{MHz} \bmod -$ ulation was determined by observing the quantum fluctuations in the radiation from a $600^{\circ}$ blackbody.

A 1-m Czerny-Turner monochromator was used to analyze the spectral distribution of the spontaneous radiation propagating at angles of up to 1.3 mrad relative to the magnet axis. A heliumcooled germanium bolometer was used with the monochromator to detect the spontaneous radiation.

Observation of the spontaneous radiation and the gain included measurement of (1) the spectral distribution and polarization of the spontaneous radiation, (2) the magnitude of the gain, and (3) the dependence of the gain on the electron energy, the polarization of the stimulating radiation fields, the electron current, the magnetic field, and the optical power density.

According to theory, the wavelengths for emission and absorption are given by

$$
\lambda=\frac{\lambda_{q}}{2 \gamma^{2}}\left[1+\left(\frac{1}{2 \pi}\right)^{2} \frac{\lambda_{q}{ }^{2} r_{0}}{m c^{2}} B^{2}\right]\left(1 \pm \frac{h \nu}{\gamma m c^{2}}\right),
$$

where $r_{0}$ is the classical electron radius $(\mathrm{cm})$, $\gamma m c^{2}$ is the electron energy (ergs), $\lambda_{q}$ is the magnet period $(\mathrm{cm})$, and $B$ is the transverse magnetic field (G). Equation (1) is for a helical magnetic field and for radiation parallel to the electron beam axis. The equation is correct to lowest or $\operatorname{der}$ in $1 / \gamma$ and $h \nu / \gamma m c^{2}$. No harmonics are present in the spectrum for radiation along the axis. Polarization is determined by the helix symmetry.

The minimum theoretical linewidth for spontaneous emission is established by the length of the magnet. For the magnet in the experiment the minimum $1 / e$ half-linewidth $(\Delta \nu / \nu)$ is $0.3 \%$. The broadening due to the finite length is homogeneous. There are also inhomogeneous effects, including the spread in energy and angular divergence of the electron beam and the variation in magnetic field over the cross section of the beam.

Figure 2(a) indicates the observed dependence on the electron energy of the spontaneous power at $10.6 \mu \mathrm{m}$. The monochromator was set at 10.6 $\mu \mathrm{m}$ while the electron energy from the superconducting accelerator was swept through a range of approximately $2 \%$ in the vicinity of $24 \mathrm{MeV}$. The resolution of the monochromator was $0.2 \%$. The
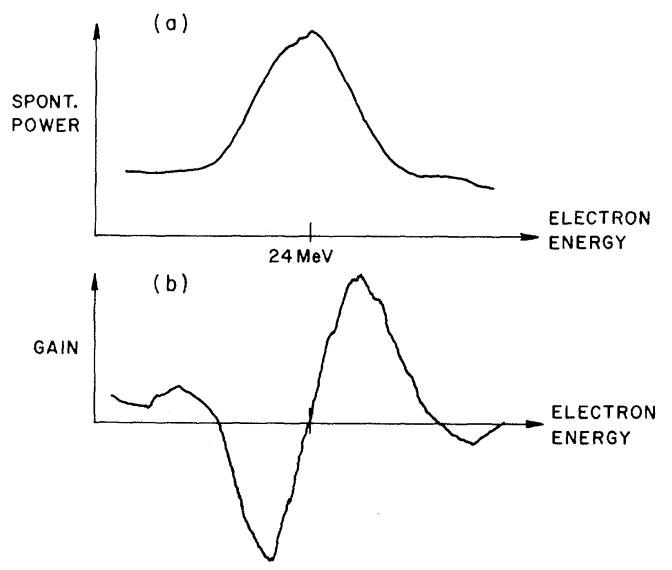

FIG. 2. (a) The spontaneous power at $10.6 \mu \mathrm{m}$ as a function of the electron energy. (b) The amplitude and phase of the modulation imposed on the $10.6-\mu \mathrm{m}$ optical radiation from the $\mathrm{CO}_{2}$ laser. Amplification corresponds to a positive signal. The instantaneous peak gain attained a value of $7 \%$ per pass. The helix field amplitude was $2.4 \mathrm{kG}$ and the instantaneous peak electron current was $70 \mathrm{~mA}$. The electron energy was swept through a small range in the vicinity of $24 \mathrm{MeV}$. The full width in energy (half-width in wavelength) at the $1 / e$ points in (a) is $0.4 \%$. The power density of the $10.6-\mu \mathrm{m}$ radiation in (b) was $1.4 \times 10^{5} \mathrm{~W} / \mathrm{cm}^{2}$.

$1 / e$ half-width of the spectrum $(\Delta \nu / \nu)$ is $0.4 \%$. The linewidth is close to the theoretical minimum. No harmonics were observed. The magnetic field was $2.4 \mathrm{kG}$. The instantaneous spontaneous power radiated into the $5 \times 10^{-6}-\mathrm{sr}$ acceptance of the detection system was $4 \times 10^{-6} \mathrm{~W}$ at an instantaneous peak current of $70 \mathrm{~mA}$. The radiation was right-circularly polarized.

Figure 2(b) indicates the dependence of the gain on electron energy. The energy scale is the same as for Figure 2(a). The trace shows the phase and amplitude of the $1300-\mathrm{MHz}$ modulation imposed on the $10.6-\mu \mathrm{m}$ optical radiation from the $\mathrm{CO}_{2}$ laser. This signal is proportional to the time-averaged gain (or absorption) per pass。The instantaneous peak gain is equal to the time-averaged gain divided by the electron-beam duty cycle, i.e., the ratio of the electron bunch length to the bunch period $(0.56 \%)$. The instantaneous peak gain per pass in Fig. 2(b) reaches 7\%. The instantaneous peak current was $70 \mathrm{~mA}$.

Figures 2(a) and 2(b) illustrate the correlation between the gain and the line shape for spontaneous emission. Note that (1) the gain falls to zero at the peak of the spontaneous spectrum, (2) there is a net gain on the high-energy side and net absorption on the low-energy side of the line-shape for spontaneous emission, and (3) the maximum 
values of gain and absorption occur at the points of inflection of the spontaneous spectrum.

Figure 2(b) was made using right-circular polarization. The measured gain was zero for leftcircular polarization. The observed gain for linear polarization was half the gain for right-circular polarization. The magnitude of the gain was observed to be a linear function of the electron current over a range of 5 to $70 \mathrm{~mA}$.

Finally, the gain was measured at optical power densities ranging from 100 to $1.4 \times 10^{5} \mathrm{~W} / \mathrm{cm}^{2}$.
The magnitude of the gain and the dependence of the gain on the electron energy were observed to be independent of the optical power density over this range. The stimulated power radiated by the electrons reached $4 \times 10^{3} \mathrm{~W}$ in these measurements. This power was $10^{9}$ times larger than the spontaneous power measured with the same electron current and corresponds to an energy loss of $60 \mathrm{keV}$ per electron in passage through the helix.

According to Madey, Schwettman, and Fairbank $^{2}$ the gain due to stimulated bremsstrahlung is given by



where $\rho_{e}$ is the density of electrons in the electron beam $\left(\mathrm{cm}^{-3}\right) ; \lambda$ and $\nu$ are the operating wavelength and frequency (cm and $\mathrm{Hz}$, respectively); $\Delta \nu$ is the 1/e half-linewidth for spontaneous emission $(\mathrm{Hz})$; $\delta \nu$ is the difference between the operating frequency and the emission line center $(\mathrm{Hz})$; and $\boldsymbol{F}_{f}$, the filling factor, is the ratio of the squares of the radii of the electron beam and the radiation field. The filling factor is unity if the electron beam radius exceeds the radius of the radiation field.

The gain equation is for a helical magnetic field and for circularly polarized radiation propagating parallel to the electron beam. Stimulated emission is possible only for that component of the stimulating radiation field which matches the polarization of the spontaneous radiation. The derivation as sumes a small signal and a weak magnetic field.

The first exponential within the braces is due to stimulated emission and the second to absorption. The line shape for stimulated emission and absorption have the same form as the line shape for spontaneous emission (a Gaussian line shape was assumed). For optical photons $2 h \nu / \gamma \boldsymbol{m} \boldsymbol{c}^{2}<<\Delta \nu / \nu$ and the difference between the two exponentials is small. The gain is then proportional to the derivative of the line shape for spontaneous emission and becomes independent of $h$. The derivative relationship is nicely illustrated by the traces in Fig. 2. The maximum available theoretical gain is

$$
G=\frac{20 \log _{10} e}{\pi}\left(\frac{1}{e \pi}\right)^{1 / 2}\left(\frac{r_{0}^{2}}{m c^{2}}\right) \lambda^{3 / 2} \lambda_{q}^{3 / 2} B^{2} \rho_{e}\left(\frac{\Delta \nu}{\nu}\right)^{-2} F_{f} \mathrm{~dB} \mathrm{~cm}^{-1} .
$$

The functional form of the gain is constrained by symmetry and dimensionality. This is important because the constraints establish scaling laws independent of the approximations used in computation. Given that the gain arises from a secondorder process and is independent of $h$, the smallsignal weak-field gain must be linear in the electron current, quadratic in the magnetic field, and vary as $\lambda^{3 / 2}$ for unity filling factor as in Eq. (3). The dependence on wavelength is modified when the filling factor is less than 1: For a fixed interaction length and optimal Guassian optics the filling factor scales as $\lambda^{-1}$ and the gain scales as $\lambda^{1 / 2}$ 。

For the experimental conditions and with the as sumption of an effective area of $0.33 \mathrm{~cm}^{2}$ for the $\mathrm{EH}_{11}$ mode, the calculated gain attains a maximum value of $5 \%$ for the $5.2-\mathrm{m}$ interaction length as opposed to $7 \%$, the observed value. The significance of the difference is not yet established; however, random errors in the measurement were negligible and the difference is believed to exceed the systematic error.

These measurements have important device implications. Lasers based on the stimulated emis sion of bremsstrahlung have a potential for continuously tunable operation at high power. The current available from existing electron machines indicates the possibility of laser operation from the infrared to the ultraviolet. The instantaneous peak current from the superconducting accelerator can be raised to the ampere level by reducing the duty cycle, and circulating peak currents in excess of $10 \mathrm{~A}$ have been obtained in electron storage rings. ${ }^{12}$

Use of a storage ring would be particularly attractive because the $\mathrm{rf}$ accelerating field for the ring would have to supply only the energy actually transformed to radiation in the periodic field. The 
overall efficiency of such a system thus would not be limited to the fraction of the electrons' energy convertible to radiation in a single pass through the interaction region. The feasibility of the idea hinges on the form of the electrons' phase-space distribution after passage through the periodic field, a subject currently under study.

An electron current of the order of 1 A at 240 $\mathrm{MeV}$ would be sufficient for laser operation at $1000 \AA$ for a $1-\mathrm{mm}^{2}$ electron-beam cross section. The measurements indicate that $0.2 \%$ of the electrons' energy can be converted to radiation in the periodic field without evidence of saturation; the corresponding power output for a $1-\mathrm{A}, 10^{8}-\mathrm{eV}$ electron beam would exceed $10^{5} \mathrm{~W}$.

We gratefully acknowledge the contributions of the Hansen Laboratory personnel and others who have assisted in this effort.

*Work supported in part by the U. S. Air Force Office of Scientific Research.

${ }^{1}$ J. M. J. Madey, J. Appl. Phys. $\underline{42}, 1906$ (1971).
${ }^{2}$ J. M. J. Madey, H. A. Schwettman, and W. M. Fairbank, IEEE Trans. Nucl. Sci. 20, 980 (1973).

${ }^{3}$ While the published Compton-scattering gain formulas can be reduced to a common functional form, the numerical factors are such that the predicted gains differ by up to two orders of magnitude.

${ }^{4}$ H. Dreicer, Phys. Fluids 7,735 (1964).

${ }^{5}$ R. H. Pantell, G. Soncini, and H. E. Puthoff, IEEE J. Quantum Electron. 4, 905 (1968).

${ }^{6}$ V. P. Sukhatme and P. A. Wolff, J. Appl. Phys. 44, 2331 (1973).

${ }^{7}$ N. Kroll, in Stanford Research Institute Technical Report No. JSR-74-1, 1975 (unpublished), p. 74.

${ }^{8} \mathrm{~J}$. L. Hirshfield, I. B. Bernstein, and J. M. Wachtel, IEEE J. Quantum Electron. 1, 237 (1965).

${ }^{9}$ V. L. Granatstein, M. Herndon, R. K. Parker, and S. P. Schlesinger, IEEE Trans. Microwave Theory Tech. 22, 1000 (1974).

${ }^{10}$ P. Sprangle, V. L. Granatstein, and L. Baker, Phys. Rev. A 12, 1697 (1975).

${ }^{11}$ R. L. Abrams, IEEE J. Quantum Electron. $\underline{8}, 838$ (1972).

${ }^{12}$ The Stanford-Princeton ring stored a peak current of $15 \mathrm{~A}$ at $300 \mathrm{MeV}$ [W. C. Barber, High Energy Physics Laboratory Report No. 350 (unpublished)]. The SPEAR ring at SLAC has stored a peak current in excess of 100 A [H. Winick, SLAC Report No. 1349 (unpublished)].

\title{
Detection of Brillouin Backscattering in Underdense Plasmas*
}

\author{
John J. Turechek and Francis F. Chen \\ Electrical Sciences and Engineering Department, University of California, Los Angeles, California 90024
} (Received 16 September 1975)

Two new results are reported: (1) verification of the finite-interaction-length threshold for stimulated Brillouin scattering in a plasma with no critical layers, and (2) use of the time-dependent Stark effect to detect ion-frequency electric fields in a plasma.

Numerous theorists ${ }^{1-4}$ have predicted that intense laser radiation $\left(\omega_{0}, \vec{k}_{0}\right)$ used to heat a plasma or implode a pellet may undergo parametric decay into an acoustic wave $\left(\omega_{i}, \vec{k}_{i}\right)$ and a backscattered light wave $\left(\omega_{1}, \vec{k}_{1}\right)$, resulting in reflection of the incident energy in the underdense, outer regions of the plasma, and thus diminishing absorption at the critical layer, where $\omega_{0}=\omega_{p} \equiv$ $\left(4 \pi n e^{2} / m\right)^{1 / 2}$ or $n=n_{c} \equiv m \omega_{0}^{2} / 4 \pi e^{2}$. This process of stimulated Brillouin scattering (SBS) has a lower threshold ${ }^{1}$ and higher nonlinear saturation ${ }^{2}$ than the competing decay into a backscattered wave and an electron plasma wave (stimulated Raman scattering). The practical threshold depends on the finite interaction length ${ }^{3}$ and the plasma inhomogeneity. ${ }^{4}$ Recent high-power measurements ${ }^{5}$ on solid targets indicate an increase in backscattered power with incident power $I_{0}$ up to $10^{15}$ $\mathrm{W} / \mathrm{cm}^{2}$, but a decrease thereafter; the latter has been attributed to a decrease in plasma scale length with intensity. ${ }^{6}$ Because solid-target experiments are complicated by plasma formation and expansion and by reflection and absorptive parametric processes occurring at the critical $\left(\omega_{0}=\omega_{p}\right)$ and quarter-critical $\left(\omega_{0}=2 \omega_{p}\right)$ layers, we have designed an experiment to test the basic finite-length linear SBS theory under conditions in which the plasma is independently created and is everywhere underdense $\left(n<n_{c} / 4\right.$ or $\left.\omega_{0}>2 \omega_{p}\right)$. Our previously reported observations ${ }^{7}$ have been confirmed by direct detection of the low-frequency field by means of the time-dependent Stark ef- 